\title{
Micromechanisms of kinking in rigid-rod polymer fibres
}

\author{
D. C. MARTIN*, E. L. THOMAS \\ Polymer Science and Engineering, The University of Massachusetts at Amherst, Amherst, \\ MA 01003, USA
}

The tensile strengths of fibres of the rigid-rod polymers poly (paraphenylene benzobisthiazole) (PBZT) and poly (paraphenylene benzobisoxazole) (PBZO) are excellent, and therefore are of particular interest for high-performance structural applications. However, these fibres are a factor of ten weaker in compression, with failure occurring by strain localization in welldefined kink bands. Here, we study the morphology of PBZT and PBZO kink bands in detail, in order to help elucidate the molecular mechanisms involved in this deformation process. We found that the typical dimensions of a kink in the direction of the fibre axis $(\sim 30 \mathrm{~nm})$ were smaller than the length of an average PBZT or PBZO molecule $(100 \mathrm{~nm})$. Also, the boundary between the kinked and unkinked regions was well-defined. Low-dose, high-resolution electron microscopy (HREM) of the kink interior revealed local, high-angle changes in chain orientation, indicative of covalent bond bending or breaking. The kink boundaries exhibit "sharp" or "smooth" features which seem to be related to the local tensile or compressive nature of the stress field. A model for kink nucleation and propagation in terms of partial dislocations is presented and discussed. A stress analysis using this model has been developed, and comparison with experimental data suggests that kinks tend to propagate towards regions of higher compressive stress. This observation is interpreted in terms of dislocation pinning (in areas of hydrostatic tension) and the nucleation of dislocation pairs (in areas of hydrostatic compression) due to the asymmetric nature of the intermolecular energy potential. Finally, practical methods for improving compressive strength based on these mechanistic insights are proposed.

\section{Introduction}

The tensile moduli and strengths of rigid-rod polymer fibres poly(paraphenylene benzobisthiazole) (PBZT) and poly(paraphenylene benzobisoxazole) (PBZO) are excellent. Theoretical predictions of PBZT and PBZO modulus have been obtained using semi-empirical quantum mechanical (minimum neglect of differential overlap) methods $[1,2]$. The theoretically predicted moduli of trans-PBZT and cis-PBZO are 605 and 730 $\mathrm{GPa}$, respectively [2]. Values nearly $50 \%$ of this theoretically predicted modulus have now been obtained experimentally [3]. Tensile strengths up to 4.9 $\mathrm{GPa}$ for PBZO and 4.2 GPa for PBZT have been observed [3, 4]. However, both PBZO and PBZT fibres are about a factor of ten weaker in compression, with failure occurring by strain localization into kink bands.

The compressive strength problem has become a severe limitation for the application of rigid-rod polymers in structural components. Hence, a deeper understanding of the molecular motions involved in kinking is a critical concern for future success in the design and processing of high-strength, high-modulus polymer fibres.

This paper presents a study of the morphology of kink bands in rigid-rod polymer fibres using optical microscopy (OM), dark-field (DF) and bright-field (BF) transmission electron microscopy, selected-area electron diffraction (SAED), and high-resolution electron microscopy (HREM). Elsewhere we investigate the thermodynamics of kinking by quantitative measurements of kinking in fibres during known amounts of compressive plastic deformation [5]. We discuss a model for kinking which involves the nucleation and growth of a kink across a fibre and describe the molecular motions involved in this deformation process. The kink band is envisioned to be a region of shear deformation separated from the undeformed part of the sample by high-angle axial chain rotation grain boundaries and partial edge dislocations. The information and insight about the molecular mechanisms and energetics of kinking obtained by this

\footnotetext{
* Present address: Materials Science and Engineering, The University of Michigan, H. H. Dow Building, Ann Arbor, MI 48109-2136, USA. ${ }^{\ddagger}$ Present address: Materials Science and Engineering, Massachusetts Institute of Technology, Room 13-5066, Cambridge, MA 02139, USA.
} 
study should prove valuable to those interested in improving the limited compressive strength of these materials.

\section{Background}

\subsection{Kinking}

The concept of kinking as a deformation mechanism in crystalline metals was introduced by Orowan [6] Both deformation twinning and kinking involve local shear within an originally undeformed sample. Kinking is distinct from deformation twinning, however, in that the material inside the sheared zone need not be a mirror image of the undeformed structure.

Orowan proposed a geometric relationship describing the likely angles for kinking based on the argument that the angle of rotation should preserve the lateral interactions of atoms across glide planes. Orowan's paper concerned kinks which had formed completely across the sample and did not elaborate on how such a deformation structure might nucleate and subsequently grow in an initially undeformed sample.

Frank and Stroh developed a theory of kinking which considered the stability of a lenticular shaped kink in an isotropic, continuous, linearly elastic medium [7]. The boundary of the kink was envisioned to be formed from a number of edge dislocations. The energy of the kink was rationalized in terms of the macroscopic stress field of the dislocations, a microscopic stress field characteristic of the dislocation cores, and the energy released during the shear deformation involved in kinking. Frank and Stroh [7] predicted that for a given applied shear stress there was a critical size $L$ for kink stability. Kinks longer than $L$ could reduce the energy of the system by growing. The model predicts that the shear stress $\tau$ required to make a kink of size $L$ propagate in a medium of shear modulus $\boldsymbol{G}$ is given by

$$
\tau=G(\gamma \boldsymbol{b} / 2 L)^{1 / 2}
$$

where $\boldsymbol{b}$ is the Burgers vector of the dislocations along the kink boundary and $\gamma$ is the shear strain within the kink. Although this analysis treats only the energetic stability of kinks which have somehow already formed, it is possible to imagine that there will always be some "effective" kink nucleus size which is determined by local defects and other structural fluctuations.

Argon developed a theory for the initiation of kinks in oriented composites [8]. His analysis considered the stress necessary to induce a shear collapse instability. The instability occurs because a compressive stress along the orientation direction causes locally misaligned elements to experience a relative shear stress which depends on the misorientation angle, $\varphi_{0}$. The model calculated the stress necessary to propagate a region of shear collapse across the sample. The change in elastic energy near the kink-collapse nucleus was modelled with a pair of edge dislocations. At the onset of the instability, Argon predicted that the stress would be given simply by

$$
\sigma_{\mathrm{c}}=\tau_{\mathrm{s}} / \varphi_{0}
$$

where $\tau_{\mathrm{s}}$ is the plastic shear strength of the matrix.

\subsection{Kinking in polymers}

Kink band formation is a general observation in the compressive failure of oriented polymers. Zaukelies [9] was the first to document kinking during compression of highly oriented nylon 6,6 and nylon 6,10 fibres and interpreted his results in terms of specific slip systems based on intramolecular bonding and close packed directions in the polymer unit cell. Observations of singly oriented fibres showed that the kinks made a uniform angle with the orientation direction but with a random distribution about the fibre axis. Although Zaukelies did not consider the questions of kink nucleation and growth, his results suggest that the nucleation of a kink specifies the angle of kinking which then grows by forcing crystallites to deform cooperatively in a manner which preserves the initial orientation of the deformation band.

Seto and Tajima [10] studied kink bands in oriented polyethylene (PE). Again, kinks were found to form at characteristic angles. The angle of kinking was a function of prior heat treatment as well as the temperature of deformation. Robertson [11] found that kinks form easily by compression of oriented crystalline polymers ( $\mathrm{PE}$ and isotactic polypropylene $\mathrm{PP}$ ), but did not form in oriented glassy polymers. Shigematsu et al. [12] studied further the morphology of kinks in oriented PE and were able to distinguish between the contributions due to uniform $c$-axis shear and intercrystalline slip by measuring both the angle of chain axis rotation as well as the resulting orientation of crystalline lamellae within the kink. It was concluded that both mechanisms must be involved in the deformation process and that the relative contributions of each might depend on deformation temperature.

Attenburrow and Bassett [13] investigated kinking during a study of the compliances and failure modes of chain-extended PE and found that the angle between the chain axis and lamellae within the kink was consistent with a uniform $c$-axis shear deformation. The uniformity of shear within the kink band as compared to conventional PE was rationalized in terms of the decreased shear resistance of the lamellar crystals in the chain-extended material.

The largest known polymer single crystals (up to $1 \mathrm{~mm}$ size or larger) are prepared from solid-state polymerization. Typical examples include polymeric sulphur nitride $(\mathrm{SN})_{\mathrm{x}}$ and polymerized diacetylenes. Because of their unique method of synthesis, such single-crystal polymers have quite different microstructures than polymers prepared by more traditional synthetic and processing routes.

Young and Baughman [14] studied shear-induced martensitic phase transformations in polymeric sulphur nitride $(\mathrm{SN})_{\mathrm{x}}$ and found that a conventional deformation twinning model explained well the geometry of the process. Petermann and Schultz [15] observed that there was a considerable amount of voiding and fibrillation within $(\mathrm{SN})_{\mathbf{x}}$ kink bands. Petermann and Schultz developed a model involving the nucleation and growth of kinks through the action of defects moving from "sources" to "sinks" corresponding to local regions of tension and compression, 
respectively. These defects were presumed to represent regions of local internal conformational changes within the polymer chains. Such a mechanism does not seem likely to explain successfully the origin of PBZO and PBZT kinks, because internal conformational changes of these molecules are not likely given the fully extended chain structure.

Deformation twinning has been useful for describing the geometry of local deformation zones in single crystals of poly(diacetylene) [16-18]. Young et al. [16] examined deformation twins in cleaved crystals of poly(diacetylene) by SEM. The twinning planes were determined from the observed angle of deformation and the known crystallographic index of the crystal cleavage face.

It was noted by Young et al. $[16,17]$ that if the boundary of a deformation twin is not strictly parallel to the crystallographic twinning plane, then the twin boundary is necessarily incoherent, requiring a distribution of dislocations to accommodate this deviation. It was proposed that the boundary of a lenticular-shaped deformation twin can be envisioned as a number of edge dislocations separated by chain tilt grain boundaries. The stresses introduced into the material by such a dislocation array were correlated with cracks parallel to the $\left[\begin{array}{ll}0 & 0\end{array}\right]$ direction which were often seen in the poly(diacetylene) matrix near deformation twins.

The morphology of kinks in a semi-rigid extendedchain polymer fibre poly(paraphenylene terepthalamide) (PPTA) was studied by Takahashi et al. [19]. It was found that kinking occurs primarily by (200) [0 01 1] slip corresponding to shear between hydrogenbonded planes of molecules. It was also observed that there was non uniformity of strain within the kink band, as well as sharp $(\sim 50 \mathrm{~nm})$ boundaries between the kink and the undeformed fibre. Because of the cylindrically symmetric orientation of the hydrogenbonded planes in PPTA, (200) [001] slip leads to helical kinks which wind their way along the fibre [20].

In PPTA, most of the tensile strength of the fibre is maintained after kinks have been introduced [21]. This suggests that bond bending or reorientation is involved at the kink boundary rather than chain breakage. Tanner et al. [22] have postulated that the kinking process in PPTA can be explained by a trans-cis conformational change of an individual PPTA molecule [22]. Such a local conformational change may very well be involved in formation of the PPTA kink boundary and for determining PPTA kink boundary angles which are of relatively low energy. However, this mechanism says little about the cooperative nature of the deformation process. Likewise, it does not explain how kinks can grow at specified angles even when the local orientation of the crystallites is such that easy trans-cis conformational changes are not possible to accommodate the strain. Finally, there are no such easy conformational changes in the rigid polymers PBZT and PBZO.

Kinks have long been known to be characteristic of compressive failure in rigid-rod polymers [23] and have also been seen in PBZT-epoxy composites [24].
In SEM studies of PBZT [25] and PBZO [26] evidence for kink bands is often seen on fibre surfaces. Inspection of these images showed that the kinks often occur in bundles, making it difficult to distinguish between single and multiple kink bands. The kinks were laterally broad (as large as the sample) and the boundaries between kinked and unkinked fibre were sharp to the resolution limit of the SEM (approximately $20 \mathrm{~nm}$ ). Sometimes small, apparently individual kinks were noted which were limited in axial extent (as small as $200 \mathrm{~nm}$ ). At times, abrupt surface features indicative of large, local plastic deformation and the lateral, outward "extrusion" of material were seen.

A theoretical model for the onset of kink formation in rigid-rod polymer fibres in terms of an elastic buckling instability has been presented [27]. This model predicts a correlation of compressive strength with the longitudinal shear modulus of the fibre, a trend which has experimental support from data for several high-performance fibre materials including PBZT, PPTA, and graphitized carbon fibres [28]. Allen has investigated the stress dependence of the torsional modulus of several polymer fibres and finds, in general, that it increases with applied tension, suggesting an instability in compression when the applied stress would cause the torsional modulus to become equal to zero [29].

DeTeresa et al. [28] comment that the model proposed by Argon (Equation 2) does not seem consistent with the observation that the compressive strengths of PBZT and Kevlar are not particularly sensitive to the overall fibre orientation [30]. Also, the Argon model predicts a rapid reduction in compressive strength for small increases in misorientation. This sensitivity is not observed experimentally in compressive strength tests of fibre composites with different amounts of misorientation [31]. However, because kink initiation is a local process, it is still possible that it is not the overall orientation but the fibre orientation distribution that is important. Careful tests of the relationship between compressive strength and the details of molecular orientation distributions have yet to be performed.

From these studies, it is possible to summarize several consistent features about the morphology of kinks in oriented polymers. The kinks are usually laterally broad (at times as wide as the sample diameter) yet limited in axial extent (less than $2 \mu \mathrm{m}$ ). The interface between the kinked and unkinked regions is relatively sharp (approximately $50 \mathrm{~nm}$ ), but detailed information about deformation close to the kink boundary and within the kink itself has been limited in all of the past studies by the resolution of the characterization techniques employed.

The morphology of the rigid-rod polymers PBZT and PBZO in the solid state is reviewed elsewhere [5, 32]. Essentially, the structure consists of molecules which are highly oriented in the direction of the fibre, but experience a large amount of axial shift disorder. The lateral ordering increases with tensioned heat treatment, resulting in discrete crystallites with a dimension of the order of $20 \mathrm{~nm}$. PBZO exhibits more three-dimensional ordering than PBZT, as evidenced 
by the development of off-axis reflections on upper order layer lines in wide-angle X-ray scattering (WAXS) and selected-area electron diffraction (SAED). The orientation, size, shape, and internal perfection of PBZO crystallites is described elsewhere in detail [33].

In this work we examine systematically the ultrastructure of kinks in the rigid-rod polymer fibres PBZT and PBZO. We wished to determine how the nature of molecular packing in these materials influenced kink morphology. Also, we hoped to understand better how materials comprised of highly oriented, completely stiff molecules could generate deformation zones in which there was a great deal of plastic strain. In the following sections we investigate the molecular mechanisms of deformation which accompany compressive failure in rigid-rod polymer fibres. In particular, our use of HREM provides a powerful tool for obtaining previously unavailable information about the molecular level structure near and within kink bands.

\section{Experimental procedure}

The fibres used in this study were heat-treated PBZT (Aftech II) and PBZO in the as-spun (AS), heat treated at $600{ }^{\circ} \mathrm{C}$ (HT 600), and heat treated at $665^{\circ} \mathrm{C}$ (HT 665) states. Samples could be kinked in one of three ways. Kinks may sometimes form simply from sample manipulation. Many kinks may be intentionally introduced due to uniform compression during thermoplastic matrix shrinkage (for example, nylon crystallized from formic acid solution) [34]. Kinks may also be introduced by floating whole fibres in water and exposing the suspension to a Branson Sonifer 185 cell disrupter $(20 \mathrm{kHz}, 25 \mathrm{~W}$ oscillation for $5 \mathrm{~min}$ ).

The morphology of the variously kinked fibres was investigated by optical microscopy (OM), bright-field (BF) and dark-field (DF) transmission electron microscopy (TEM), SAED, and high-resolution electron microscopy (HREM). Samples for electron microscopy were prepared by the detachment replication technique described elsewhere [5].

For HREM, a $200 \mathrm{kV}$ JEOL $2000 \mathrm{FX}$ transmission electron microscope equipped with a beam blanking device, Gatan video system and minimum dose system (MDS) was used. Suitable areas for imaging were found by searching with a low-intensity beam in diffraction mode. After final focusing on an adjacent area, the sample was exposed to a electron dose equal to less than one-third of the total end-point dose, $\left(J_{c}\right)$, of the equatorial refiections. Typical experimental conditions were a magnification of $\times 10^{5}$, screen current of $10 \mathrm{pA} \mathrm{cm}^{-2}$, and exposure time of $0.5 \mathrm{~s}$. This was sufficient to result in an optical density of 0.5 on Kodak SO-163 electron image film developed for 12 min in full strength D-19. Details of these procedures are given elsewhere [5].

\section{Results}

Fig. 1 shows optical micrographs of kinks in various heat-treated PBZO fibres. The kinks shown in Fig. 1

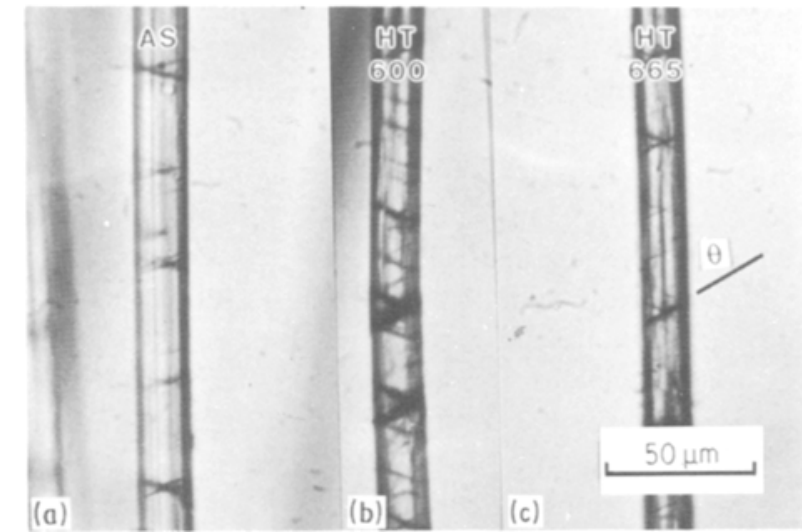

Figure I Optical micrographs of kinks in PBZO fibres: (a) as-spun, (b) HT 600, and (c) HT 665.

TABLE I Angle of kink with respect to fibre axis from optical microscopy

\begin{tabular}{lll}
\hline Fibre & Angle (deg) & $\sigma_{\mathrm{d}}$ \\
\hline PBZT & 72.6 & 7.8 \\
PBZO as-spun & 75.4 & 2.6 \\
PBZO HT 600 & 68.6 & 4.8 \\
PBZO HT 665 & 66.4 & 4.0 \\
\hline
\end{tabular}

were produced by sample manipulation. During OM observation, it was observed that by rotating the fibre and changing the focus of the microscope, conditions could be found where a particular kink would appear sharp and distinct, presumably because the direction of shear within the kink was then parallel to the focal plane. The kinks thus imaged in the OM were at a well-defined angle to the fibre axis. Table I shows the angle, $\Theta$, of the kink boundary with respect to the fibre axis for PBZO as a function of heat treatment. These numbers were obtained by averaging over at least 25 kinks for each material. There seems to be a slight tendency for the angle of kinking in PBZO to decrease with heat treatment.

Kinks were often observed during TEM investigation of PBZT and PBZO fibre fragments. All of the kinks observed by TEM were found to have at least one lateral edge extending completely to a free surface of the fibre fragment. This suggests that kinks initiate at the fibre surface and then grow in towards the body of the fibre. Fig. 2 shows a equatorial DF image of a kink in PBZO HT 600. Bright areas in this image correspond to regions of the sample (PBZO crystallites) that are scattering electrons into the objective aperture. From the position of the aperture in this experiment, these bright regions correspond to areas which have good lateral packing and are oriented in the fibre direction. By moving the objective aperture or rotating the sample during DF imaging it was confirmed that the material within kink bands was misoriented but remained crystalline. This was also confirmed by SAED and by HREM imaging. Note that in Fig. 2 the kink is dark, indicating that the molecules within the kink band have undergone a large change in orientation. 


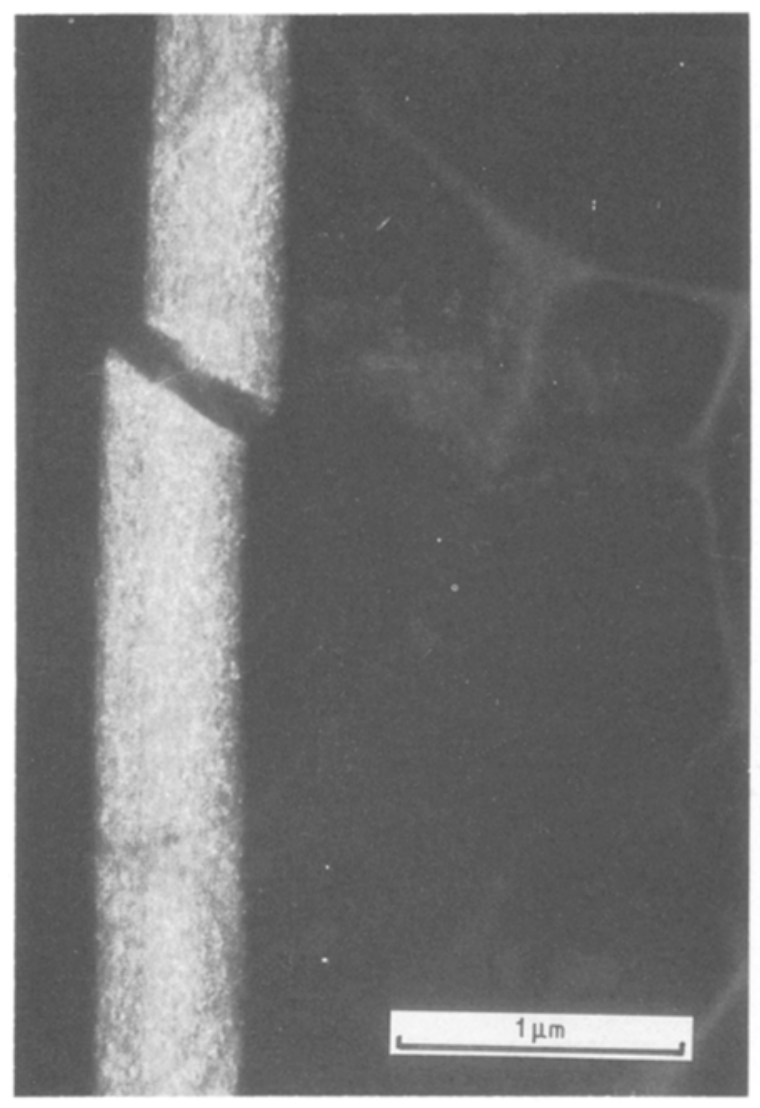

Figure 2 Equatorial dark-field image of a kink in a PBZO HT 600 fibre fragment. The boundaries between the kink and the undeformed fibre are well-defined and localized.

From Fig. 2, note the sharp boundaries $(10 \mathrm{~nm}$ or less) between the kink and the undeformed fibre. Viewing the image from the side shows that the upper boundary is approximately straight (fluctuations $\pm 20 \mathrm{~nm}$ from a line) across the fibre whereas the lower boundary is more meandering (fluctuations $\pm 60 \mathrm{~nm}$ ). Most of the kinks observed by EM had propagated all the way across the fibre fragment as in Fig. 2. Fig. 3 shows another equatorial DF of PBZO HT 600 in which there is a kink which has not propagated across the full thickness of the fibre fragment. The size of this kink in the direction of the fibre axis is about $30-50 \mathrm{~nm}$. There is also a kink "bundle" at the bottom of this image where several kinks exist in a local region. Note that the kinks seem to adopt preferred orientations with respect to the fibre axis. Although the geometry of kinks seen in fibre fragments is difficult to determine because of the "detachment" step during sample preparation, the angle measured here $\left(67^{\circ}\right)$ corresponds well with that observed at lower resolution by $\mathrm{OM}$.

Careful inspection of the kink which has propagated part way across the fibre shows different features for the upper boundary (where the local deformation is away from the centre of the fibre fragment) and lower boundary (where the local deformation is toward the centre of the fibre fragment). The change in contrast at the upper boundary is sharp (changing from bright to dark in $6 \mathrm{~nm}$ ), whereas the change in contrast at the lower boundary is more diffuse (chang-

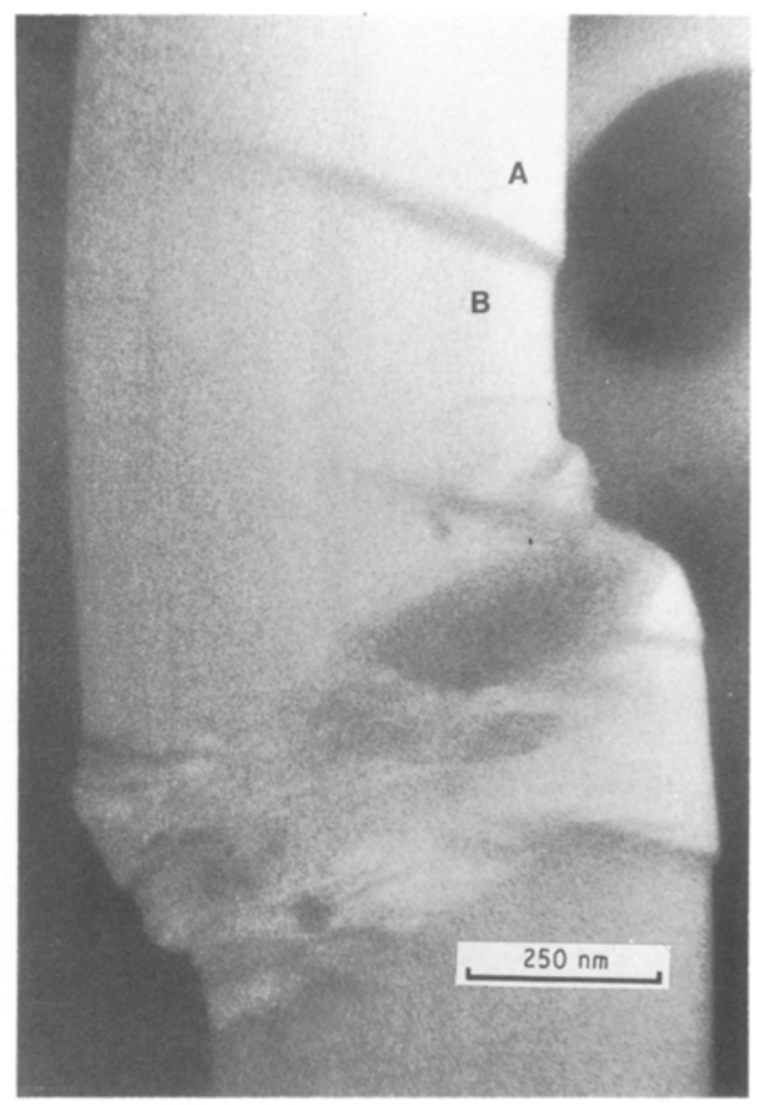

Figure 3 Equatorial dark-field image of a kink in PBZO HT 600 in which a kink has not propagated all of the way across the fibre fragment. Near the bottom of the figure is a "bundle" in which several kinks have formed in the same region. The DF image contrast shows that the upper boundary of the isolated kink (A) is "sharp" (bending in $6 \mathrm{~nm}$ ), while the lower boundary (B) is more "diffuse" (bending in $15 \mathrm{~nm}$ ). The size of the kink in the axial direction $(30-50 \mathrm{~nm})$ is significantly less than the average size of a PBZO molecule (100 nm).

ing from bright to dark in $15 \mathrm{~nm}$ ). Because the contrast in this image is related to the local orientation of the PBZO molecules, these results imply that the misorientation angle as a function of distance along the fibre is more discontinuous at the upper kink boundary than at the lower.

Another characteristic type of kinking which was identified was an S-shaped configuration. One such instance of this type of kink in PBZO HT 600 is shown in Fig. 4. The common features of these S-shaped kinks include a stripe of axially oriented material in the centre of the kink (A) as well as extremely localized bending as evinced by $10-20 \mathrm{~nm}$ bands of equatorial DF contrast near the edge of the fibre (B). This observation suggests that not only is it possible to bend the rigid-rod polymer chains, but they may be bent several times before failure. The plastic strain estimated from this particular image is greater than $\varepsilon$ $=0.3$.

Kink bands in PBZT and PBZO usually appeared to be of uniform density. However, fibres of PBZO were sometimes observed to exhibit fibrillation and void formation inside the kink band. This is similar to that seen in $(\mathrm{SN})_{\mathrm{x}}$ by Schultz and Petermann [35]. A BF example of such a fibrillated kink is shown in Fig. 


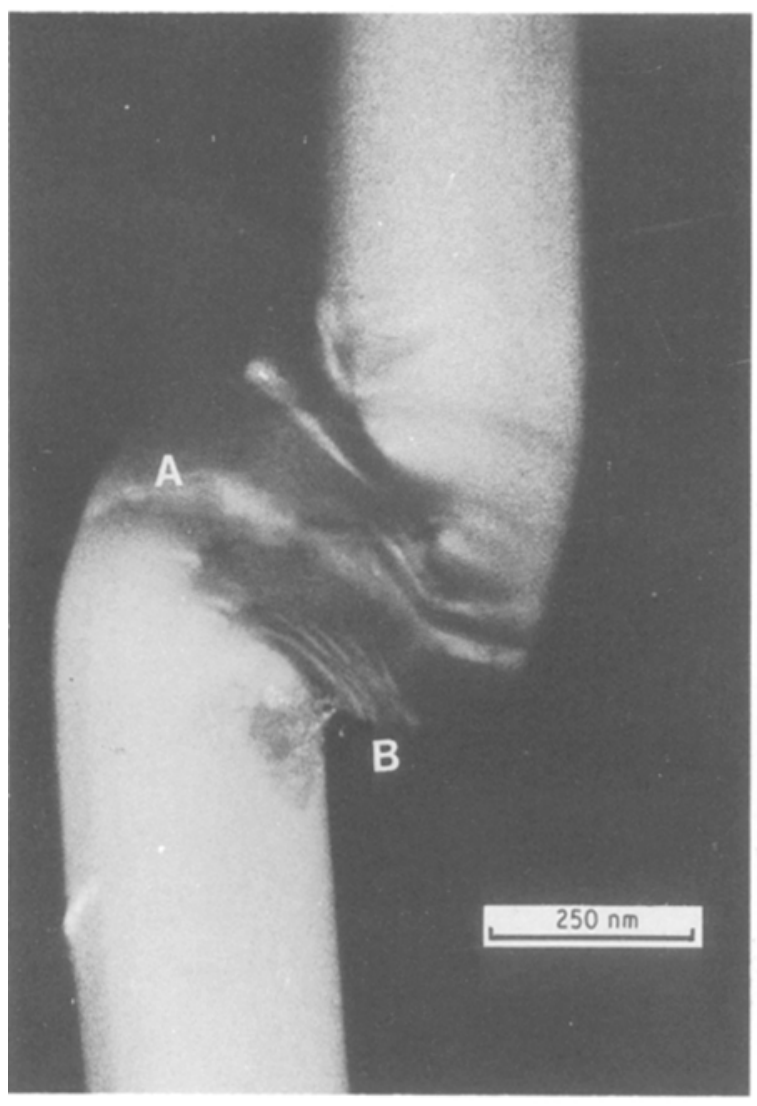

Figure 4 Equatorial dark-field image of an S-shaped kink in PBZO HT 600 . The characteristic features of these types of kinks include a stripe of axially oriented material (A) as well as localized buckling near the fibre edge $(B)$

5. Both scattering and phase-contrast effects are present in the image at this resolution. The formation of elongated voids within the kink is easily noted. Also, the boundary region between areas which can be considered as belonging to the kinked and unkinked regions of the fibre is remarkably sharp (less than $0.5 \mathrm{~nm}$ ).

Fig. 6 is a tracing of a HREM lattice image of a kink band in PBZT. The orientation of the diffracting crystallites may be determined precisely and is shown with $20 \mathrm{~nm}$ long dashes as a function of position. Again, there is evidence for a sharp variation in contrast near the boundary of the kink where the deformation is away from the centre of the fibre (A), while the boundary corresponding to deformation towards the fibre is more diffuse (B). In this BF image there are contrast changes due to mass thickness effects, suggesting material "extrusion" or "bulging" at the upper surface of the kink where the deformation is away from the body of the fibre.

Fig. 7 is an HREM lattice image of a kink band in PBZO HT 665. The edge of the fibre fragment is to the right as in the previous micrographs. The arrows on top and bottom indicate the molecular direction in the undeformed regions of fibre on either side of the kink. Several striking features of the deformation process are visible. The local heterogeneity of the kinking strain is obvious. A tilt boundary corresponding to an abrupt change in molecular orientation $\left(38^{\circ}\right.$ over a distance of about $0.5 \mathrm{~nm}$ ) is seen (A). Other features such as a local curvature of the PBZO lattice are

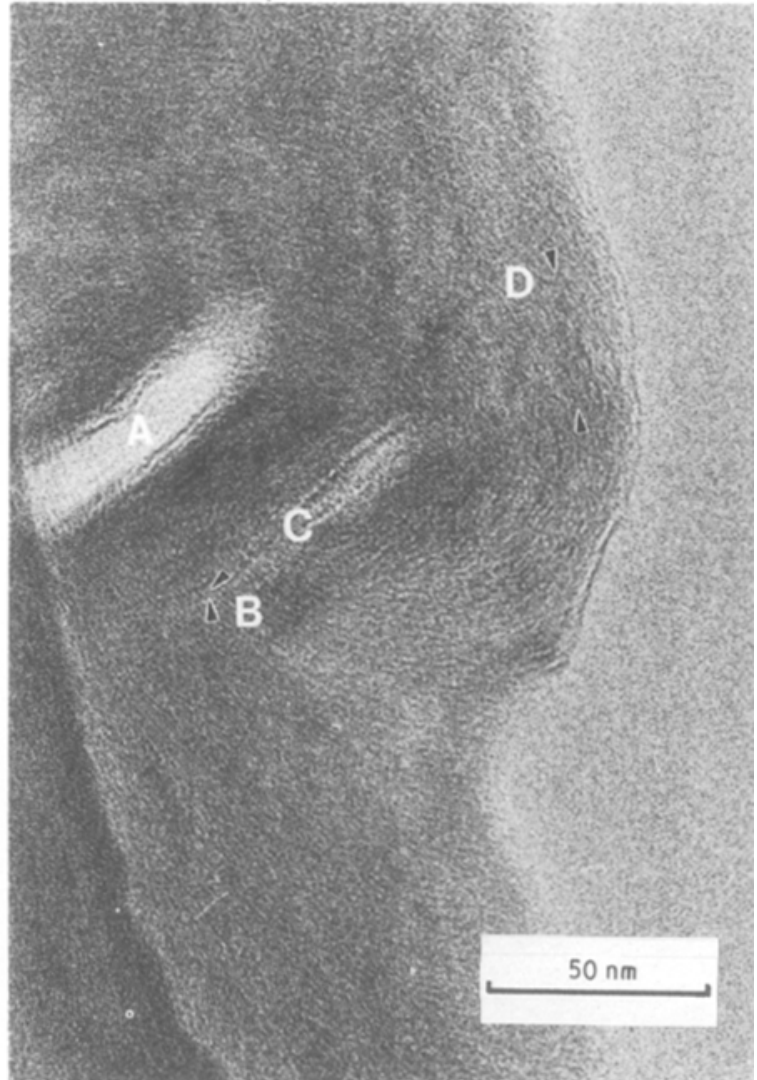

Figure 5 Fibrillated kink in PBZO HT 665. Elongated voids can be seen within the kink (A). The boundary region between kinked and unkinked material $(B)$ is very sharp (less than $0.5 \mathrm{~nm}$ ). Irradiation caused a slight opening of the kink during the exposure (C). Careful inspection reveals lattice resolution of the $0.55 \mathrm{~nm}(100)$ planes near the edge of the fibre (D)

apparent (B). Finally, note the abrupt change and sharp contrast variation at the upper edge of the kink (C). This feature is again indicative of a local change in the thickness or diffraction conditions, indicative of a lateral failure causing material to be "extruded" at the upper boundary.

From these and similar images it is possible to measure certain geometrical features pertaining to the kink deformation process. Fig. 8 shows a plot of the number of occurrences of kinks in PBZO with a particular dimension in the direction of the fibre axis. Note that there is a strong tendency to form kinks in the range $20-30 \mathrm{~nm}$. These TEM results suggest that only the smallest features in SEM images of fibre surfaces are due to individual kinks, and that kinks observed in OM are due to kinks or bundles which have grown to a substantial size in order to cause significant scattering of light.

\section{Discussion}

In this section we first review the implications of our morphological observations in terms of the geometry of kinking in rigid polymers. We then discuss a model for estimating the stress distribution around a propagating kink in terms of partial dislocations, and interpret our experimental data in terms of this analysis. Finally, we present a detailed molecular mechanism for the initiation and propagation of kink bands in rigid-rod polymers. 


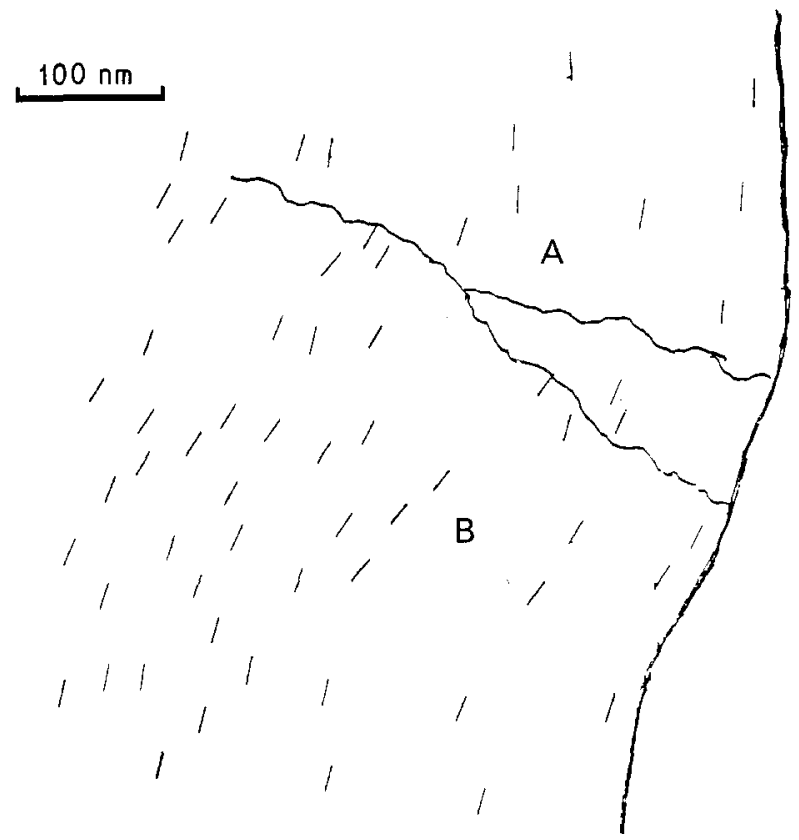

Figure 6 Sketch of a HREM image of PBZT showing the orientation of $0.59(100)$ or $0.36(010)$ lattice fringes as a function of position near a kink. The edge of the fibre as well as sharp surface features are drawn as solid lines. Sharp contrast features were near kink boundaries where the local direction of deformation was away from the body of the fibre (A), whereas the boundaries corresponding to deformation toward the fibre body were more smooth and diffuse (B).

Our results indicate that kinks tend to be significantly smaller in the direction of the fibre axis $(20-30 \mathrm{~nm})$ than the length of an average molecule $(100-200 \mathrm{~nm})$. Sharp, high-angle tilt boundaries between adjacent crystals have been directly imaged within kink bands by HREM. These experimental observations reveal that a local cooperative bending and/or breaking of covalently bonded polymer molecules is involved in kinking. The strain within the kink is inhomogeneous; local crystallite misorientation, fibrillation, and bending of the lattice have all been detected.

The fact that there is a characteristic angle of kinking suggests an analogy of between kinking in these fibres and deformation twinning in atomic crystals. We now further examine this relationship in terms of the unit cell of PBZT and PBZO.

In rigid rod crystals the easy slip systems are those which involve sliding parallel to the molecular axis. These slip planes are of general index $\{h k 0\}$. Likewise, in the kinking of fibres the direction of slip is along the fibre direction. It follows that the shearing planes belong in the $\{h k 0\}$ family. It is also apparent that the direction of shear and the boundary of the kink are not parallel to [001], but from the observed angle of kinking (Table I), it is not possible to index the shear direction and kink boundary plane with small integer crystallographic indices.

The nature of the stress field which is present around a kink which has not propagated fully across a fibre may be envisioned by considering the accommodation strain required to incorporate a kink with non-parallel edges. Fig. 9 shows a fibre before and

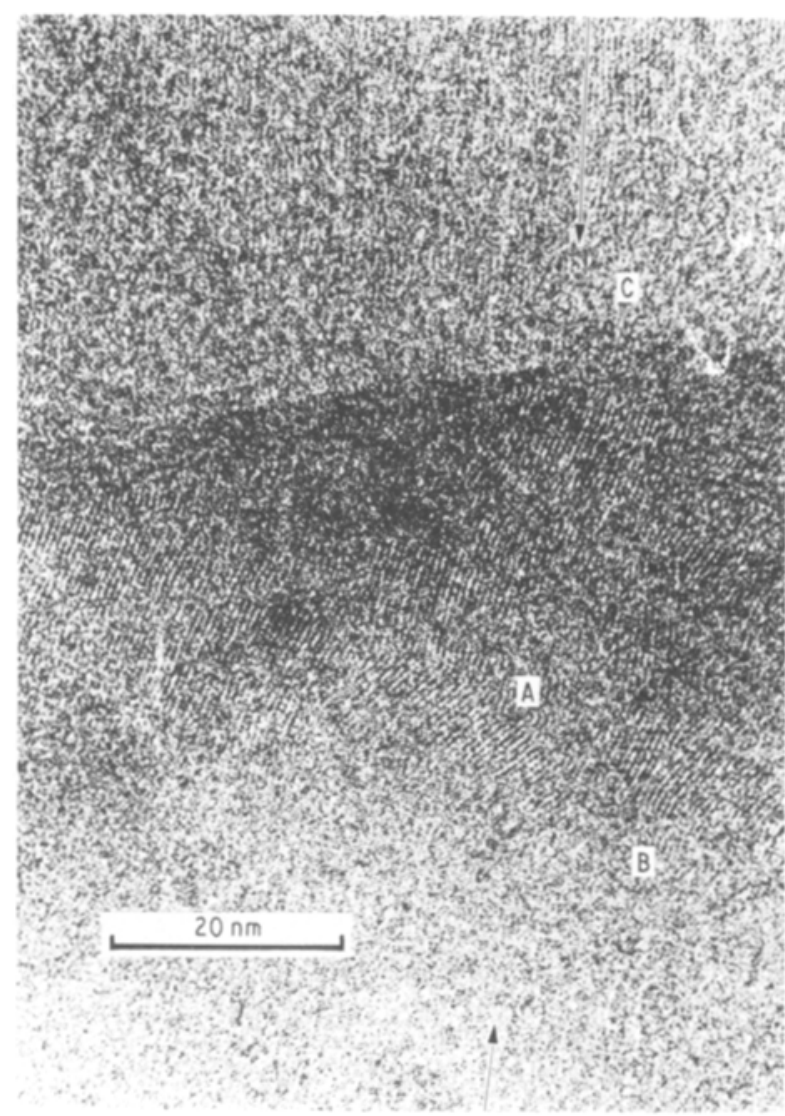

Figure 7 HREM image of a kink in PBZO HT 665. The heterogeneous nature of deformation within the kink is evident. Sharp, highangle tilt boundaries are seen (A). Evidence for local bending of the PBZO lattice is noted (B). The nearest edge of the fibre fragment is to the right. Sharp variations in mass thickness contrast are seen on the upper boundary where the direction of deformation is toward the edge of the fibre fragment (C).

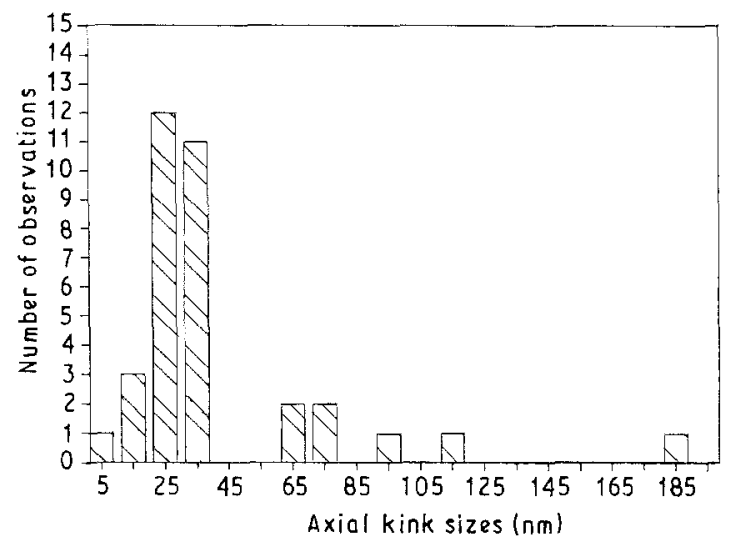

Figure 8 Histogram of the frequency of observation of a kink of a given axial size in DF images of PBZO. There is a strong tendency to form kinks in the $20-30 \mathrm{~nm}$ size range.

after a wedge of material undergoes a uniform shear. Note that the material near the edge of the wedge which deforms away from the body of the fibre is dilated and therefore should be in relative tension. The lower edge of the wedge is compressed and therefore would be in relative compression.

It is possible to describe the strain field around a propagating kink or deformation twin in terms of high-angle tilt boundaries and partial dislocations. The dislocations involved in kinking or 

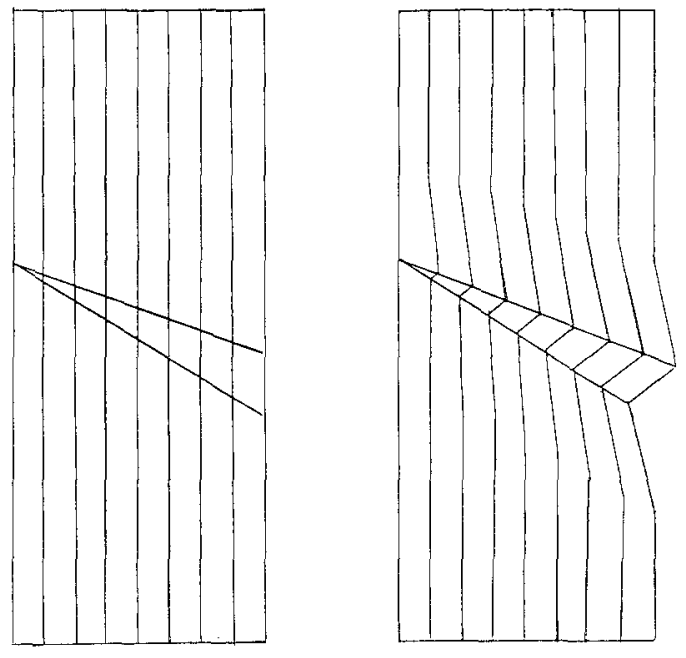

Figure 9 Schematic diagram illustrating the geometry of kinking. This drawing shows the case in which the crystalline packing is perfectly maintained after deformation and the boundaries of the kink band are not parallel to one another. In order to accommodate the kink band the upper boundary must deform away from the interior of the fibre, whereas the lower boundary must deform towards the fibre. This implies that the upper boundary would be in relative tension, whereas the lower boundary would be in relative compression.

twinning are termed "partial" because their Burgers vectors do not necessarily correspond to a full translation of the lattice. Also, they deform but do not disrupt the continuity of the lattice. The use of such dislocations to accommodate the strains near twins was described first by Vladimirskii [36]. Frank and van der Merwe [37] described these as "dislocations of second order". The Burgers vector of the dislocation lies in the twin boundary, which is also the glide plane. Olson and Cohen [38] have suggested the name "coherency glide dislocations" to recognize the important fact that the lattice is deformed yet retains its connectivity. A similar dislocation is the partial edge dislocation attributed to Shockley which occurs at a stacking fault termination within a crystal $[39,40]$. A model of this type of dislocation is shown in Fig. 10. The role of dislocations in twinning and their relationship to twin geometry has been described by Kosevich and Boiko [40] and by Kosevich [41].

Fig. 11 shows a model of a kink containing tilt boundaries and several partial dislocations. The tilt boundaries separate the region of shear deformation from the undeformed part of the sample. The local edge or screw character of the dislocations will depend on whether the direction of shear deformation is locally perpendicular or parallel to the kink boundary, respectively. Fig. 11 shows a view of the kink from the side so that the direction of shear is perpendicular to the kink boundary, thus these dislocations are edge in character.

The dislocations represent the local strain dilation above and compression below the kink front necessary to accommodate the shear deformation. The Burgers vectors of the edge dislocations are parallel to the kink boundary and have a magnitude equal to the strain necessary to return the deformed region back to its originally undeformed configuration. This relates

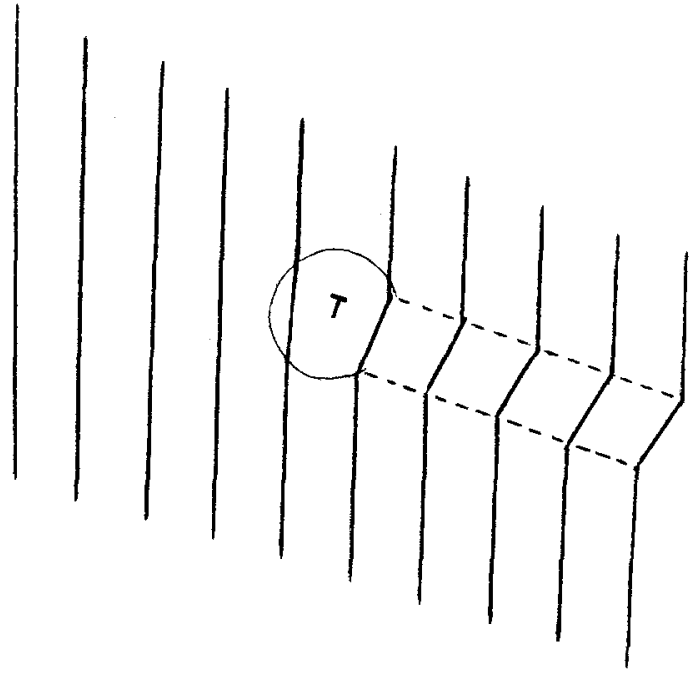

Figure 10 Idealized model of the partial dislocations and tilt boundaries involved in the kinking of rigid-rod polymers. The dark lines represent the backbones of the molecules, and the dashed lines the high-angle tilt boundaries separating the kinked and unkinked regions of the fibre. Adapted from Fig. 5.2 of [39].

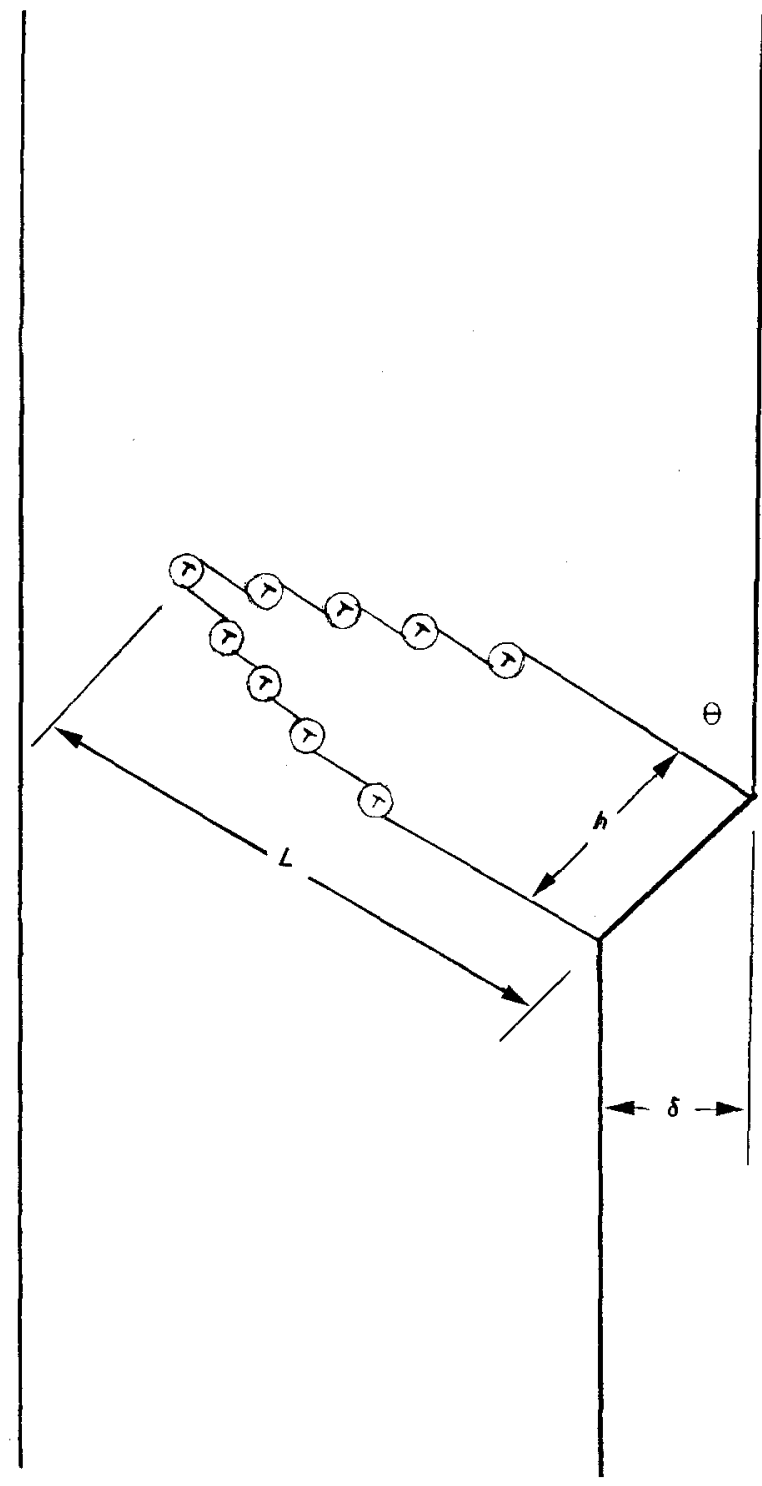

Figure 11 Schematic drawing of the partial dislocation model of kink formation and propagation. The mechanism involves the formation of a bundle of dislocations which propagate across the sample. The number and position of the dislocations in a given kink can be determined from the experimentally observed kink geometry if the Burgers vector is known or if one is assumed. 
to the amount of shear induced as a dislocation passes an originally undeformed area. As shown in Fig. 9, the accommodation strain field around the dislocation bundle is expected to be dilatational on top of the bundle (as drawn) and compressional on the bottom.

Using this model, kink formation in rigid-rod polymer fibres may be considered as the nucleation and subsequent propagation of a bundle of partial dislocations across the body of the fibre. In the model shown in Figs 10 and 11, the dislocations are separated by sharp, coherent tilt boundaries across which the rigidrod polymers undergo a large, local change in orientation. This deformation will require a local bending and/or breaking of a rigid-rod polymer molecule. This model is similar to that proposed by Young et al. [16-18] for deformation twinning in poly(diacetylene). However, the partial dislocations involved in kinking of axially disordered fibres do not seem as well-formed and localized as they are in materials with large crystals.

As first noted by Keith and Passaglia [42] the motion of a dislocation through a polymer crystal in a direction which is not parallel to the orientation of the chains results in a plane of bent molecules. Further deformation by this same mechanism is unlikely because multiple slip would require chain scission. However, it would be possible for the plasticity to continue by having the dislocations reverse direction, causing a kink to propagate back across the fibre in a sense opposite to the original motion. Such a mechanism might explain the origin of "bundles" (Fig. 3) and "Sshaped" kinks (Fig. 4).

An important point to recognize is that it is not necessary to have a well-defined crystal lattice in order to have a dislocation. The Burgers vector of a dislocation in a continuous substance is defined by the path integral of the elastic displacement around the dislocation direction [43]. Dislocations can be used to describe any inhomogeneous strain distribution, but this representation is not necessarily unique [39]. Future work should clarify the applicability of dislocation models in partially disordered systems and compare these dislocations with those in systems with much more developed order.

By analogy with deformation twinning, the strains characterizing the shape of the kink at the surface are indicative of the number of dislocations present within the sample interior, as long as the dislocation geometry is known or assumed [40]. From Fig. 11, the amount of lateral strain is $\delta$ and the size of the kink in the direction of the fibre is $h$. The number of dislocations involved in making such a kink is given by $h=N \boldsymbol{a}$ and $\delta=N \boldsymbol{b}$, where $\boldsymbol{a}$ is the projection of the Burgers' vector in the direction of the fibre axis and $b$ is the projection of the Burgers vector perpendicular to the fibre axis. From the kink in Fig. 4 we measure $\delta$ $=14 \mathrm{~nm}$ and $h=32 \mathrm{~nm}$. This gives a ratio $h / \delta=\boldsymbol{a} / \boldsymbol{b}$ $=2.3$. Because of the axially disordered structure of PBZO and PBZT, the choice of a characteristic lattice dimension is somewhat arbitrary. However, a useful and well defined characteristic length along the axis of the fibre is $d_{001}=1.2 \mathrm{~nm}$, the monomer repeat distance. If this value is used for $\boldsymbol{a}$, then $\boldsymbol{b}=0.52 \mathrm{~nm}$. This is close to the lateral spacing between neighbouring molecules, namely (for PBZO) $d_{200}=0.55 \mathrm{~nm}$. Using these values as the characteristic size for the Burgers vectors comprising the kink gives $N=25$. Hence, the kink in Fig. 3 may be modelled as a propagating bundle of 25 partial dislocations with $a=1.2 \mathrm{~nm}$ and $b=0.55 \mathrm{~nm}$.

It is possible to use this dislocation model to estimate the form of the stress field around a propagating kink band. Using a larger value for the Burgers vector enables us to rescale the problem and analyse data such as the dark-field image in Fig. 3 at a lower resolution. Fig. 12a shows the partial kink from Fig. 3 and the positions of the dislocations determined with an extended Burgers vector of $\boldsymbol{a}^{\prime}=5 \boldsymbol{a}=6.2 \mathrm{~nm}$. Whereas before there were 25 dislocations with the choice of $\boldsymbol{a}=1.24 \mathrm{~nm}$, there are a net of 5 large dislocations with $\boldsymbol{a}^{\prime}=6.2 \mathrm{~nm}$ in this kink. The positions of these dislocations were determined by finding the intersections of lines perpendicular to the edge of the kink with the kink boundary (Fig. 12b). During this exercise it was found that the kink had propagated down and into the fibre far enough for it to be necessary to include a second set of dislocations of opposite sign at the bottom of the kink. Hence, by choosing $\boldsymbol{a}^{\prime}=6.2 \mathrm{~nm}$ for the Burgers vector, the outline of the kink in Fig. 12a can be described by 11 edge dislocations with the Burgers vectors pointing toward the interior of the fibre on the upper side of the kink, and 6 edge dislocations with the Burgers vectors pointing toward the outer surface of the fibre on the lower side of the kink.

The solution to stress field around an edge dislocation in an isotropic medium is well known [43]. The stress depends on position of the dislocation, the shear modulus $G$, the magnitude of the Burgers vector $\boldsymbol{b}$, and Poisson's ratio $v$. The magnitude of the stress is proportional to $(1 / r)$, where $r$ is the distance away from the dislocation.

This solution is appropriate only for isotropic, linearly elastic media with localized dislocations. Obviously, the highly anisotropic nature of PBZO and PBZT makes it necessary to seek a rigorous solution to the stress and strain distribution near a kink in these materials with anisotropic dislocation theory [44]. Rigorous solutions to problems in anisotropic elasticity theory may lead to results which are nonintuitive [45]. However, our interest here is in establishing the general form of the stress field around a propagating kink in terms of a simple dislocation model. This naive approach provides a helpful understanding of the micromechanisms of kink nucleation and propagation. The success of this method provides an impetus for future studies where more realistic aspects of known material behaviour can be incorporated.

The stress field around the dislocation array for the kink in Fig. 12a was solved numerically by assuming linear elastic behaviour. The stress field of the sample was determined by the linear superposition of the individual stress fields generated by each dislocation. The result of this calculation is shown in Fig. 12c where the hydrostatic component of the stress field $\left(\sigma_{x x}+\sigma_{y y}\right)$ is shown as a function of position around 

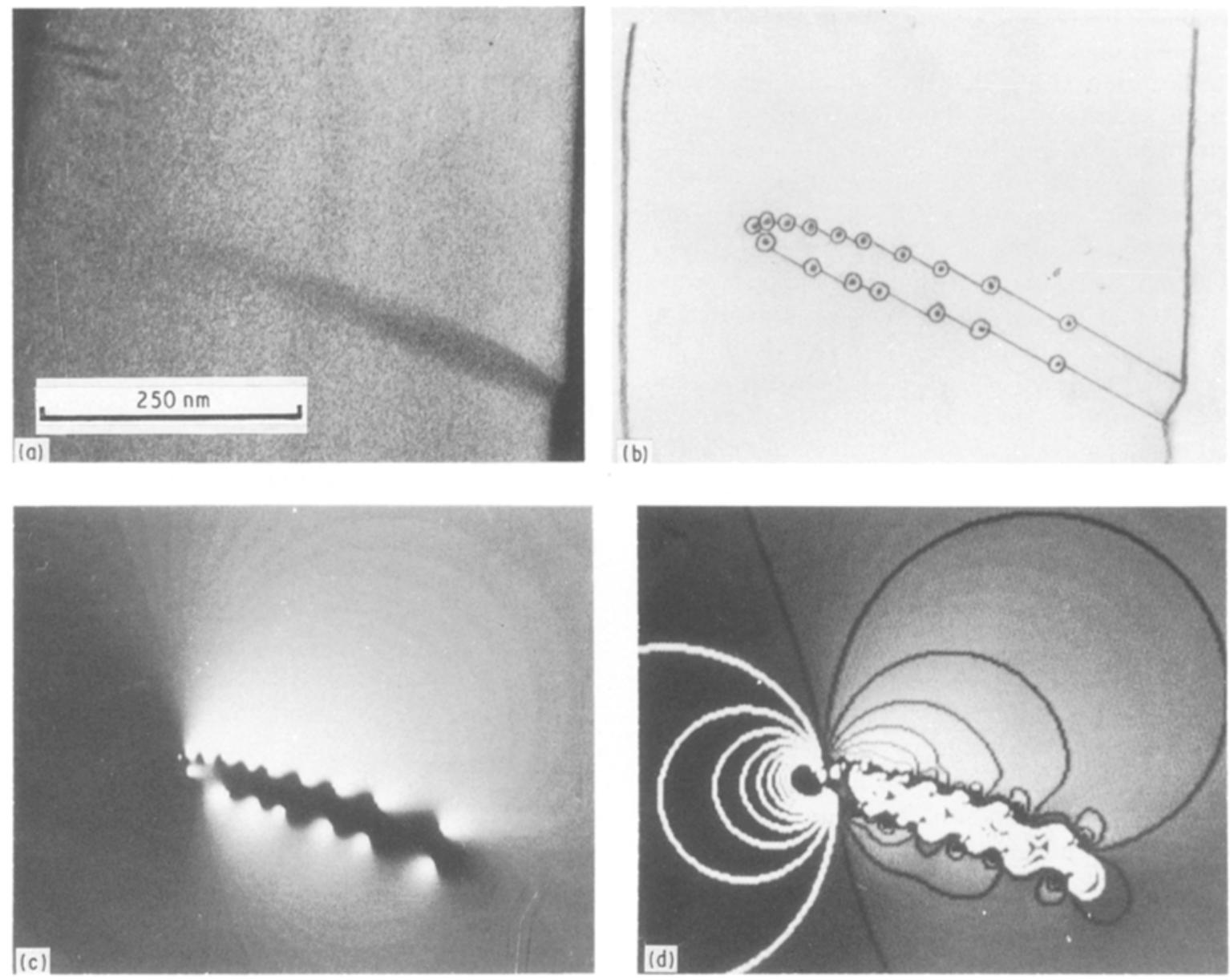

Figure 12 Dislocation stress model. (a) Detail from Fig. 4 showing a kink which had not propagated completely across the fibre. (b) Positions of the dislocations determined using $b=6.2 \mathrm{~nm}$. (c) The hydrostatic component of the stress field around the kink using the dislocation model. Hydrostatic tension is shown in white and compression in black. (d) Iso-stress contour lines. Note that the kink is apparently curving toward the region of higher compressive stress.

the kink. The image is dark where the material is predicted to be in local hydrostatic compression and light where there is local hydrostatic tension. Contours of constant hydrostatic pressure are shown in Fig. 12d.

It is apparent that from Fig. $12 \mathrm{c}$ and $\mathrm{d}$ that there is a region of hydrostatic tension on the upper side of the kink and hydrostatic compression on the lower side of the kink. The kink has migrated noticeably toward the compressive side, curving away from its initial trajectory.

The "sharp" and "smooth" image contrast features of the kink previously mentioned can now be rationalized in terms of this stress distribution around the kink and the asymmetric nature of the intermolecular potential function. In compression, the energy simply increases as the repulsive forces act to resist strain, and there is no instability. However, in tension an instability occurs at a point when the effective modulus (the curvature of the energy-position function) becomes zero.

If such an instability does occur, then the dislocations defining the boundary of the deformation band might become pinned on the tensile boundary when adjacent molecules split apart in the lateral direction. The kink would then grow by propagation towards the compressive side. This might involve the nuclea- tion of pairs of dislocations of opposite sign, similar to the mechanism employed in the kink nucleation model of Argon [8]. Further support for this mode of kink propagation can be found by a more careful analysis of the morphology of kinks in other materials. Indications of growth toward the compressive direction can be seen in macroscopic kinks of carbon fibre composites observed by OM [46]. Evidence for lateral splitting can be seen near deformation twins of poly(diacetylene) $[16,17]$. Lateral splitting in $\mathrm{PBZO}$ is evident within the kink band in Fig. 6, and is sometimes visible by SEM [26].

It does not seem likely that the kinking dislocations move smoothly across the fibre during the deformation process. The glide planes involved are non-crystallographic and oriented at an oblique angle to the covalently bonded fibre axis. Therefore, it seems reasonable that dislocation glide on these planes will be severely hindered. A more realistic model than uniform glide might be the irregular nucleation of dislocation "packets" in the region preceding the kink boundary. As noted earlier, the curvature of the kink toward the compressive side suggests that these dislocations preferentially nucleate in pairs of opposite sign on the compressive side of the propagating kink front. This mechanism of growth might explain why some fully grown kinks show one kink boundary which is sharp 
and well formed, while the other is more irregular (see for example, Fig. 2).

Besides the problem of restricted dislocation glide, a further complication is the non-uniformity of shear strain within the kink. Fig. 7 shows that while crystallinity is maintained after kinking, the strain within the kink is very non-uniform. We interpret this to mean that not every plane within the kink band undergoes the same amount of shear with respect to its neighbours. In other words, the shear within the kink is "blocky" rather than "fine" shear. This is certainly the case in the PBZO fibre which was seen to exhibit fibrillation within the kink band (Fig. 5). This nonuniformity of shear strain is reasonable given the polycrystalline nature and small crystallite size which leads to a high abundance of lateral and axial grainboundary defects. The nature of grain-boundary defects in extended-chain polymers is discussed elsewhere $[5,47]$. Axial chain invariant (ACI) grain boundaries will involve the intertwining of polymer molecules and therefore may be particularly resistant to shear deformation.

For rigid-rod polymers in tension the tensile breaking stress is about $4 \mathrm{GPa}$ and the modulus is about $400 \mathrm{GPa}$, or $\sigma_{1} / E=10^{-2}$. If we assume that the shear stress, $\tau$, and shear modulus, $G$, which control kinking have the same ratio, we obtain a value of the critical kink dimension, $L$, from the Frank and Stroh model equal to $1.7 \mu \mathrm{m}$ using $b=0.5 \mathrm{~nm}$ and the shear strain $\gamma$, which is given by $2 \operatorname{cotan}(\Theta)$ [48]. The corresponding width of the kink is obtained from the Frank and Stroh model by the relation $w=L \tau / G \gamma$, which in our case would be $24 \mathrm{~nm}$. From the histogram of kink dimensions (Fig. 9), we see that this is in reasonable agreement with our experimental observations.

The validity of dislocation models in understanding the plastic deformation behaviour of disordered systems has been discussed by Argon [49]. In crystalline solids, plastic deformation occurs by the familiar mechanism of dislocation motion. Dislocation sources usually are not widely and uniformly distributed and therefore the deformation always becomes localized. The mechanism of plasticity in disordered media such as metallic glasses is proposed to involve a set of local shear transformations having a distribution of free energy barriers which depend on the free volume distribution. The difference is that structural disorder severely hinders easy dislocation motion; therefore the material can support higher loads and becomes more "activated", that is, it is possible to nucleate new sources of deformation more uniformly throughout the material than in the crystalline case.

In kinking of rigid-rod polymer fibres we seem to be somewhere between these two extremes. The orientation of the molecule along the fibre provides for easy slip along the [001] direction, but the high axial disorder, fibre symmetry, and small crystallite size mean that the local defect concentration is much higher than in materials with larger crystals. These local constraints serve to provide an increased resistance to deformation that enables the nucleation of new dislocations at the propagating kink front. Hence, we have a deformation process that becomes localized because of the ability of the fibre to slip along its axis and form a kink band, but the propagation of the kink band is hindered by the disorder perpendicular to the fibre axis and therefore involves the activation of new sources of deformation at the leading edge of the kink.

To summarize, a model for the micromechanisms of kink deformation in rigid-rod polymer fibres is presented. First, there is an initiation step which involves the nucleation of a bundle of partial dislocations which delineate a region of shear deformation which extends to the edge of the fibre. The driving force for the nucleation of these dislocations is the reduction in matrix compressive strain energy caused by the shear strain along the kink boundaries. Once this nucleus is formed, the angle of kinking is defined. Subsequent propagation of the kink involves the cooperative rotation of subsegments of neighbouring polymer molecules as the kink front moves past. Instabilities occur on the tensile side of the kink front which impair dislocation motion causing lateral splitting and void formation. This promotes the propagation of the kink toward the compressive side of the deformation zone. This growth involves the nucleation of new pairs of edge dislocations with opposite sign.

With this model of the molecular motions involved in compressive deformation of polymer fibres in mind, it is useful to consider possible routes for improving the compressive strength of these materials. We reconfirm the conclusion of the buckling instability model which says it is important to improve the shear modulus. We also find that modifications which make it harder for kinks to initiate, such as surface hardening, would be useful. Such a scheme might involve the cross-linking or partial graphitization or oxidation of the sample surface.

Because we have established that local, sharp bending of molecules is involved in kinking, molecular structures such as "ladders" and "sheets" which are even more difficult to bend than PBZT and PBZO molecules are of interest. Also, modifications which increase the amount of energy required to shear the material within the kink will decrease the amount of energy available for kink propagation. An example might be a molecule which becomes somehow "twisted" or "intertwined" with its neighbours. This might be why nature has designed collagen fibres with a triple-stranded twisted helix structure of the protein molecules in the tropocollagen subunit [50].

The axial disorder and small crystallite size in these materials suggests that the inherent kink nucleus size is probably already quite small. Dramatic improvements in crystallite size and ordering will be useful if they can serve to improve lateral interactions, but the formation of very large PBZT or PBZO crystals may facilitate easy slip and therefore make kinking easier. Similar arguments hold for crystal shape. Long, thin crystals should restrict the glide of dislocations across the fibre but will facilitate axial slip, whereas short, oblate crystals should restrict axial slip but may make it easier for lateral dislocation glide.

Similar arguments can be made about other schemes for trying to hinder dislocation motion across the fibre and therefore disrupt kink propagation. A 
possibility might involve the placement of a second phase or perhaps local cross-linking to act as dislocation obstacles. Such microstructural modifications will be successful only if they do not serve as kink initiation sites.

Another problem with large PBZT and PBZO crystallites is that this might promote the preferential segregation of chain ends to the crystal boundaries. This would have a negative impact on mechanical properties due to the presence of well-defined flaws which would serve as kink nucleation sites in compression and as pre-cracks in tension. This argument can be extended further to consider the importance of the molecular weight distribution. If the polydispersity of the molecules were small, then any tendency for chain ends to segregate would be repeated all the way down the fibre axis. A broad distribution in molecular length makes it possible for these local flaws to be more randomly distributed and therefore of lesser importance.

Finally, it is worth mentioning that the formation of kink bands is not necessarily a "bad" characteristic. Kinks serve to absorb a substantial amount of energy and therefore enable high-performance fibres to be well suited for applications where toughness is a primary concern. It should be kept in mind that modifications to improve compressive strength will be most useful if they can increase the stress required for kink initiation yet still allow the deformation to precede by kinking without causing fracture once the instability occurs. In this way, one will obtain a material which is simultaneously strong, stiff, and tough.

\section{Conclusions}

We have established by low-dose HREM imaging that sharp, localized bending occurs during kinking in rigid-rod polymer fibres. The characteristic dimension of kinks in the direction of the molecular axis is often much smaller than the average size of each molecule. We have observed large angle changes in molecular orientation $\left(40^{\circ}\right)$ at very sharp $(0.5 \mathrm{~nm})$ tilt boundaries. We therefore conclude that local bending and/or breaking of covalent bonds is involved in the formation of kink bands in rigid-rod polymers.

A stress analysis of a propagating kink using partial dislocations indicates regions of local hydrostatic tension and compression near the kink front. The kinks exhibit "sharp" changes in orientation and material density on the tensile side of the kink, whereas the compressive side is more "diffuse". The kinks apparently have a tendency to curve as they grow, moving down toward the region of compressive stress ahead of the kink. We interpret these results in terms of lateral instabilities involving splitting failure due to the asymmetric nature of the intermolecular potential and the tensile nature of the local hydrostatic stress state.

\section{Acknowledgements}

The authors thank W. Wade Adams, Wright Patterson Air Force Base, for supplying samples and for useful conversations. DCM thanks the Shell Company for financial support in the form of a Doctoral Research Fellowship. ELT thanks the AFOSR for support through grants $85-0275$ and F49620-89-C0073.

\section{References}

1. W. W. ADAMS, P. G. LENHERT, J. J. P. STEWART, H. E. KLEI, R. K. EBY, H. JIANG and J. SMITH, Bull. Amer. Phys. Soc: 32 (1987) 780.

2. S. G. WEIRSCHKE, AFWAL-TR-88-4201 (1988).

3. W. W. ADAMS and R. K. EBY, Mater. Res. Soc. Bull. XII (8) (1987) 22.

4. H. LEDBETtER, Dow Chemical Co., USA, personal communication to W. Wade Adams, WPAFB (1987).

5. D. C. MARTIN, PhD dissertation, University of Massachusetts at Amherst (1990).

6. E. OROWAN, Nature 149 (1942) 643.

7. F. C. FRANK and A. N. STROH, Proc. Roy. Phys. Soc. $\mathbf{B 6 5}$ (1952), 811.

8. A. S. ARGON, "Fracture of Composites", in "Treatise on Materials Science and Technology," Vol. 1 (Academic Press, New York, 1972) pp. 79-114

9. D. A. ZAUKELIES, J. Appl. Phys. 33 (1962) 2797.

10. T. SETO and Y. TAJIMA, Jpn. J. Appl. Phys. 5 (1966) 534.

11. R. E. ROBERTSON, J. Polym. Sci. A-2 7 (1969) 1315.

12. K. SHIGEMATSU, K. IMADA and M. TAKAYANAGI, J. Polym. Sci. Polym. Phys. Ed. 13 (1975) 73.

13. G. E. ATTENBUR ROW and D. C. BASSETT, J. Mater. Sci. 14 (1979) 2679.

14. R. J. YOUNG and R. H. BAUGHMAN, ibid. 13 (1978) 55.

15. J. PETERMANN and J. M. SCHULTZ, ibid. 14 (1979) 891.

16. R. J. YOUNG, D. BLOOR, D. N. BATCHELDER and C. L. HUBBLE, ibid. 13 (1978) 62.

17. R. J. YOUNG, R. DULNIAK, D. N. BATCHELDER and D. BLOOR, J. Polym. Sci. Polym. Phys. Ed. 17 (1979) 1325.

18. I. M. ROBINSON, P. H. J. YEUNG, C. GALIOTIS, R. J. YOUNG and D. N. BATCHELDER, J. Mater. Sci. 21 (1986) 3440.

19. T. TAKAhashi, M. MiURA and K. SAKURI, J. Appl. Polym. Sci. 28 (1983) 579.

20. S. J. DeTERESA, S. R. ALLEN, R. J. FARRIS and R. S. PORTER, J. Mater. Sci. 19 (1984) 57.

21. S. J. DeTERESA, PhD dissertation, University of Massachusetts at Amherst (1986).

22. D. TANNER, A. K. DHINGRA and J. J. PIGLIACAMPI, J. Metals 38 (1986) 21.

23. S. R. AlleN, A. G. FILIPPOV, R. J. FARRIS, E. L. THOMAS, C. P. WONG, G. C. BERRY and E. C. CHENEVEY, Macromol. 14 (1981) 1135.

24. Y. COHEN, and E. L. THOMAS, ibid. 21 (1988) 433.

25. S. R. ALLEN, PhD dissertation, University of Massachusetts at Amherst (1983).

26. W. W. ADAMS, D. L. VEZIE and S. J. KRAUSE, AFWALTR-88-4082 (1988).

27. S. J. DeTERESA, R. S. PORTER and R. J. FARRIS, J. Mater. Sci. 20 (1985) 1645.

28. Idem, ibid. 23 (1988) 1886.

29. S. R. ALLEN, Polymer 29 (1988) 1091

30. Idem private communication (1989).

31. S. DeTERESA, private communication (1989)

32. D. C. MARTIN and E. L. THOMAS, in "The Materials Science and Engineering of Rigid-Rod Polymers", edited by W. Wade Adams, R. Eby and D. McLemore, Materials Research Society Symposium Proceedings (Materials Research Society, Pittsburg, 1989) p. 134.

33. D. C. MARTIN and E. L. THOMAS, Macromol. (1991) in press.

34. S. J. DetereSA, R, J. FAR RIS and R. S. PORTER, Polym. Compos. 3 (1982) 57.

35. J. M. SCHUltz and J. PEtermanN, Phil. Mag. $A \mathbf{4 0}$ (1979) 27.

36. K. V. VLADIMIRSKII, Zh. Eksp. Teor. Fiz. 17 (1947) 530.

37. F. C. FRANK and J. H. VAN DER MERWE, Proc. Roy. Soc. 198 (1949) 205. 
38. G. B. OLSON and M. COHEN, "Dislocation Theory of Martensitic Phase Transformations", in "Dislocations in Solids, Vol, 7, edited by F. R. N. Nabarro (North-Holland, Amsterdam, 1986) Ch. 37.

39. D. HULL, "Introduction to Dislocations", 2nd Edn (Pergamon, Oxford, 1975)

40. A. M. KOSEVICH and V. S. BOIK O, Sov. Phys. Uspekhi 14 (1971) 286.

41. A. M. KOSEVICH, "Crystal Dislocations and" the Theory of Elasticity", in "Dislocations in Solids", Vol. 1, edited by F. R. N. Nabarro (North-Holland, 1979) Ch. 1.

42. H. D. KEITH and E. PASSAGLIA, J. Res. NBS 68A (1964) 513.

43. J. P. HIRTH and J. LOTHE, "Theory of Dislocations", 2nd Edn (Wiley, New York, 1982).

44. J. W. STEEDS and J. R. WILLIS, Dislocations in Anisotropic Media", in "Dislocations in Solids", edited by F. R. N. Nabarro (North-Holland, Amsterdam, 1979) Ch. 2.
45. R. J. FAR RIS, private communication (1989).

46. A. G. EVANS and W. F. A DLER, Acta Metall. 26 (1978) 725.

47. D. C. MARTIN and E. L. THOMAS, Phil. Mag. $A(1991)$ in press.

48. R. E. REED-HILL, "Physical Metallurgy Principles", 2nd Edn, Brooks/Cole Engineering Division, (Litton Educational, Monterey, CA, 1973).

49. A. S. ARGON, "Dislocations in Non-crystalline Media?", in "Dislocation Modelling of Physical Systems, Proceedings of the International Conference", edited by M. F. Ashby, R. Bullogh, C. S. Hartley and J. P. Hirth, Gainesville, FL, 22-27 June 1980, (Pergamon Press, 1981) p. 393.

50. J. GROSS, Sci. Amer. 204 (5) (1961) 120.

\section{Received 31 October}

and accepted 19 November 1990 